\title{
Longitudinal Changes in the Corpus Callosum following Pediatric Traumatic Brain Injury
}

\author{
Trevor C. Wu ${ }^{a}$ Elisabeth A. Wilde ${ }^{d, e}$ Erin D. Bigler ${ }^{a-c}$ Xiaoqi Li ${ }^{d}$ \\ Tricia L. Merkley ${ }^{a}$ Ragini Yallampalli ${ }^{d}$ Stephen R. McCauley ${ }^{d, f}$ \\ Kathleen P. Schnelle ${ }^{d}$ Ana C. Vasquez ${ }^{d}$ Zili Chu $^{9, i}$ Gerri Hanten ${ }^{d}$ \\ Jill V. Hunter ${ }^{g, i}$ Harvey S. Levin ${ }^{d, h}$

\begin{abstract}
a Department of Psychology and ${ }^{\mathrm{b}}$ Neuroscience Center, Brigham Young University, Provo, Utah, 'Department of Psychiatry and the Utah Brain Institute, University of Utah, Salt Lake City, Utah, dPhysical Medicine and Rehabilitation Alliance of Baylor College of Medicine and the University of Texas-Houston Medical School, Departments of ${ }^{e}$ Radiology and Neurology, ${ }^{\mathrm{f}}$ Neurology and Pediatrics, ${ }^{\mathrm{g}}$ Radiology and hNeurology, Neurosurgery and Pediatrics, Baylor College of Medicine, and 'E.B. Singleton Department of Diagnostic Imaging, Texas Children's Hospital, Houston, Tex., USA
\end{abstract}

\section{Key Words}

Cognitive development • Developmental changes • Morphometry · Neurodevelopment · Traumatic brain injury • White matter • Diffusion tensor imaging

\footnotetext{
Abstract

Background: Atrophy of the corpus callosum (CC) is a documented consequence of moderate-to-severe traumatic brain injury (TBI), which has been expressed as volume loss using quantitative magnetic resonance imaging (MRI). Other advanced imaging modalities such as diffusion tensor imaging (DTI) have also detected white matter microstructural alteration following TBI in the CC. The manner and degree to which macrostructural changes such as volume and microstructural changes develop over time following pediatric $\mathrm{TBI}$, and their relation to a measure of processing speed is the focus of this longitudinal investigation. As such, DTI and volumetric changes in the CC in participants with TBI and a comparison group at approximately 3 and 18 months after injury
}

\section{KARGER}

(C) 2010 S. Karger AG, Basel

Fax +4161306 1234

E-Mail karger@karger.ch

www.karger.com
Accessible online at: www.karger.com/dne as well as their relation to processing speed were determined. Methods: Forty-eight children and adolescents aged 7-17 years who sustained either complicated mild or moderate-to-severe TBI $(n=23)$ or orthopedic injury (OI; $n=25)$ were studied. The participants underwent brain $M R I$ and were administered the Eriksen flanker task at both time points. Results: At 3 months after injury, there were significant group differences in DTI metrics in the total CC and its subregions (genu/anterior, body/central and splenium/posterior), with the TBI group demonstrating significantly lower fractional anisotropy (FA) and a higher apparent diffusion coefficient $(A D C)$ in comparison to the Ol group. These group differences were also present at 18 months after injury in all CC subregions, with lower FA and a higher ADC in the TBI group. In terms of longitudinal changes in DTI, despite the group difference in mean FA, both groups generally demonstrated a modest increase in FA over time though

T.C.W. and E.A.W. contributed equally to this study. 
this increase was only significant in the splenium/posterior subregion. Interestingly, the TBI group also generally demonstrated ADC increases from 3 to 18 months though the OI group demonstrated ADC decreases over time. Volumetrically, the group differences at 3 months were marginal for the midanterior and body/central subregions and total CC. However, by 18 months, the TBI group demonstrated a significantly decreased volume in all subregions except the splenium/posterior area relative to the OI group. Unlike the Ol group, which showed a significant volume increase in subregions of the CC over time, the TBI group demonstrated a significant and consistent volume decrease. Performance on a measure of processing speed did not differentiate the groups at either visit, and only the OI group showed significantly improved performance over time. Processing speed was related to FA in the splenium/posterior and total CC only in the TBI group on both occasions, with a stronger relation at 18 months. Conclusion: In response to TBI, macrostructural volume loss in the CC occurred over time; yet, at the microstructural level, DTI demonstrated both indicators of continued maturation and development even in the damaged CC, as well as evidence of potential degenerative change. Unlike volumetrics, which likely reflects the degree of overall neuronal loss and axonal damage, DTI may reflect some aspects of postinjury maturation and adaptation in white matter following TBI. Multimodality imaging studies may be important to further understand the long-term consequences of pediatric TBI.

Copyright $\odot 2010$ S. Karger AG, Basel

\section{Introduction}

Traumatic brain injury (TBI) is a major public health concern. According to Langlois et al. [2005], approximately 475,000 children are treated for TBI annually in the USA, with the highest rate of hospitalization in the adolescent age range $(129 / 100,000)$. Closed head trauma arising from falls and motor vehicle crashes is the most frequent cause of unintentional pediatric TBI, while penetrating brain injury accounts for less than 5\% of TBI in the pediatric population. Children and adolescents are particularly vulnerable to closed head trauma [Thurman et al., 2007], and TBI sustained at this critical developmental stage can have a profound negative impact on learning, behavior and psychological outcome [Yuan et al., 2007].

The neuropathology associated with TBI has been traditionally classified as either focal or diffuse injury. Focal injury such as cerebral contusions or intracranial hemor- rhage can be readily identified on computed tomography (CT) or magnetic resonance imaging (MRI) [Benson et al., 2007; Povlishock and Katz, 2005; Smith et al., 2003]. In contrast, diffuse injury is usually less evident on conventional imaging. Recent studies have demonstrated the sensitivity and utility of diffusion tensor imaging (DTI) in identifying and characterizing microstructural pathologies including those occurring from TBI [Alexander et al., 2007; Arfanakis et al., 2002; Ashwal et al., 2006; Chu et al., 2010; Ewing-Cobbs et al., 2006; Mayer et al., 2010; Nakayama et al., 2006; Rugg-Gunn et al., 2001; Salmond et al., 2006; Wilde et al., 2006, 2008; Wu et al., 2010; Xu et al., 2007].

DTI is based on two theoretical assumptions: water molecules in the brain are constantly moving, and the directionality and magnitude of diffusivities of water molecules are indicative of the integrity of underlying neuronal structures [Alexander et al., 2007; Mori and Zhang, 2006; Mukherjee et al., 2008]. Healthy axonal membranes constrain water molecules intracellularly and are closely compacted in tracts minimizing free water in extracellular spaces as well [Yamada et al., 2009]. The DTI technique capitalizes upon this combination of constraining water diffusivity within and around the axon where aggregate white matter tracts can be inferred by linking similar characteristics of water diffusivity [Yamada et al., 2009]. This also means that when an axon is damaged, water diffusion is altered, which can be detected by various DTI metrics including fractional anisotropy (FA) and the apparent diffusion coefficient (ADC). FA measures the degree to which diffusion occurs in one particular direction, and is indicated by a coefficient ranging from 0 to 1 , where 0 is completely random and 1 is maximally anisotropic. The ADC reflects the overall magnitude of diffusivity. A small ADC represents greater constrained diffusivity. Neuronal swelling, cytoskeleton or alterations in extracellular space secondary to injury and edema, often seen immediately following mild TBI, have been shown to disrupt normal diffusivities, resulting in increased FA and decreased ADC [Bazarian et al., 2007; Chu et al., 2010; Mayer et al., 2010; Wilde et al., 2008; Wu et al., 2010]. It is not clear whether the initial increase in FA is limited to mild TBI or whether this finding reflects the feasibility of performing DTI during the subacute phase of mild injury. In contrast, during axonal degeneration following TBI, water diffusivity increases, which reduces FA and increases the ADC [Alexander et al., 2007; Assaf et al., 1997; Belanger et al., 2007; Benson et al., 2007; Ducreus et al., 2005; Newcombe et al., 2007; Povlishock and Katz, 2005; Sidaros et al., 
2008; Wilde et al., 2008; Xu et al., 2007], which relates to histologically identified axonal pathology [MacDonald et al., 2007a, b; Wang et al., 2009].

Following TBI, several axonal pathways have robustly demonstrated altered diffusivities, including the corpus callosum (CC), anterior limb of the internal capsule, cingulum bundles, uncinate fasciculi, centrum semiovale, thalamic white matter projections, and inferior and superior longitudinal fascicule though results vary across ages and regarding the amount of time after injury and severities of injury [Arfanakis et al., 2002; Babikian et al., 2010; Bendlin et al., 2008; Huisman et al., 2004; Inglese et al., 2005; Kumar et al., 2010; Levin et al., 2008; Little et al., 2010; Nakayama et al., 2006; Niogi et al., 2008; Ptak et al., 2003; Rugg-Gunn et al., 2001; Sidaros et al., 2008; Wang et al., 2008; Wilde et al., 2006, 2008; Xu et al., 2007]. Among these pathways, the CC is selectively vulnerable where callosal damage is common in children and adolescents with TBI [Beauchamp et al., 2009; Ewing-Cobbs et al., 2008]. The long-coursing nature and the midline location adjacent to the falx cerebri make CC particularly susceptible to the traumatic axonal injury resulting from shear strain forces. Decreased FA and increased ADC [Bendlin et al., 2008; Ewing-Cobbs et al., 2008; Gorrie et al., 2001; Kraus et al., 2007; Levin et al., 2008; Wilde et al., 2006], as well as volumetric reduction [Anderson and Bigler, 1994; Benavidez et al., 1999; Kim et al., 2008; Levin et al., 1990; Tomaiuolo et al., 2004; Wilde et al., 2006] in the CC following TBI are consistently documented in children and adolescents during the subacuteto-chronic stage. However, to date, no study has systematically examined how volumetric changes in the CC relate to changes in DTI metrics in a longitudinal design.

Given the widespread interhemispheric connections that course through the $\mathrm{CC}$ and its primary function in mediating interhemispheric communication [Pannek et al., 2010], callosal pathway disruptions can have a profound impact on cognitive functioning, including the speed of information processing [Aukema et al., 2009; Biegon et al., 1994; Chepuri et al., 2002; Jokinen et al., 2007; Turken et al., 2008]. Patients with TBI often demonstrate slowed information processing on neuropsychological testing [Battistone et al., 2008; Johansson et al., 2009; Madigan et al., 2000]. In a previous cross-sectional study, we have found a significant relation between speed of processing and CC integrity as measured by DTI FA in children and adolescents with TBI at 3 months after injury [Levin et al., 2008].

In the present study, we examined longitudinal changes in CC volume and microstructural integrity from 3 to
18 months after injury in a group of children and adolescents with complicated mild and moderate-to-severe TBI and in a comparison group of children and adolescents with orthopedic injury (OI), as well as the relation between these imaging findings and speed of information processing. We hypothesized that the OI group would have higher FA and lower ADC in the total CC and its subregions (genu/anterior, body/central and splenium/ posterior) at 3 months after injury, and that these differences would become more pronounced at 18 months as a result of either the disruption of a normal developmental trajectory or continued degeneration. We anticipated that the TBI group would demonstrate slower cognitive processing speed than the OI group.

\section{Patients and Methods}

\section{Participants}

This study was approved by the institutional review boards of all institutions from which data were collected. Informed consent was obtained from each participant or his/her legal guardian as appropriate. All participants were recruited from consecutive admissions to emergency rooms at level 1 trauma centers in Houston, Dallas and Miami. All participants were fluent in English and were between the ages of 7 and 17 years at the time of study enrollment. As part of the study design, MRI and cognitive assessments were planned for 3 and 18 months after injury.

The TBI group was comprised of 23 participants ( 15 males and 8 females) between the ages of 7.8 and 17.2 years (mean: $12.9 \pm$ 3.2 years) who sustained either complicated mild or moderate-tosevere TBI. The severity of the injury was determined by the postresuscitation Glasgow Coma Scale (GCS) scores [Teasdale and Jennett, 1974]. Severe TBI was defined as a GCS score of 3-8, and moderate TBI as a score of 9-12. A complicated mild TBI was defined as a GCS score of 13-15 with acute (within $24 \mathrm{~h}$ after injury) trauma-related CT findings (e.g. contusions and hematomas). Participants with complicated mild TBI were recruited because the presence of cerebral lesions is predictive of poor cognitive outcome at 12 months after injury [Levin et al., 2008]. Given these classifications, there were 16 severe, 4 moderate and 3 complicated mild cases. Other eligibility criteria included an Abbreviated Injury Scale (Committee on Injury Scaling, 1998) score of $<4$ for body parts other than the head, and an Injury Severity Scale score of $<12$. The modal mechanisms of injury were motor vehicle or motorcycle crashes. All but 1 TBI participant were right-hand dominant.

The OI group included 25 participants $(18$ males and $7 \mathrm{fe}-$ males), ages ranging from 7.1 to 16.3 years (mean: $11.8 \pm 2.7$ years), who were hospitalized overnight, generally for upper or lower extremity bone fractures. The OI comparison group controls for risk factors that predispose individuals to injury, including preexisting behavioral problems, subtle learning disabilities and family variables. The absence of significant previous head trauma in the OI group was confirmed via a detailed developmental questionnaire administered to the parent or legal guardian, 
Table 1. Demographic and injury characteristics of TBI and OI groups

\begin{tabular}{|c|c|c|c|c|}
\hline Demographics & TBI group $(\mathrm{n}=23)$ & OI group $(n=25)$ & t value $/ \chi^{2}$ value & $\mathrm{p}$ \\
\hline Range & $7.8-17.2$ & $7.1-16.3$ & & \\
\hline Race/ethnicity distribution & $\begin{array}{l}8 \text { Caucasian, } 1 \mathrm{AA} \text {, } \\
1 \text { American Indian, } 13 \text { Hispanic }\end{array}$ & $\begin{array}{l}10 \text { Caucasian, } 7 \text { AA, } \\
6 \text { Hispanic, } 1 \text { Asian, } 1 \text { biracial }\end{array}$ & Fisher's exact & 0.026 \\
\hline Social Composite Index (z-score) & $0.05 \pm 0.91$ & $0.27 \pm 0.89$ & 0.86 & 0.394 \\
\hline Range & -1.9 to 1.4 & -1.5 to 1.7 & & \\
\hline Handedness & 22 right, 1 left & 21 right, 4 left & Fisher's exact & 0.350 \\
\hline Mechanism of injury (accident type) & $\begin{array}{l}12 \text { MVC, } 4 \text { motorcycle, } 2 \text { RVC, } \\
1 \text { bicycle, } 2 \text { falls, } 2 \text { hit by motor vehicle }\end{array}$ & $\begin{array}{l}1 \text { motorcycle, } 1 \text { RVC, } 7 \text { falls, } \\
1 \text { hit by a falling object, } 14 \text { sports/play, } 1 \text { other }\end{array}$ & Fisher's exact & $<0.0001$ \\
\hline $\begin{array}{l}\text { GCS score } \\
\text { Range }\end{array}$ & $\begin{array}{l}7.5 \pm 4.1 \\
3.0-15.0\end{array}$ & N/A & $\mathrm{N} / \mathrm{A}$ & N/A \\
\hline $\begin{array}{l}\text { Time after injury - } 3 \text { months, months } \\
\text { Range }\end{array}$ & $\begin{array}{l}4.0 \pm 0.9 \\
2.5-5.3\end{array}$ & $\begin{array}{l}4.2 \pm 1.0 \\
2.7-7.1\end{array}$ & 0.49 & 0.629 \\
\hline
\end{tabular}

Values denote means \pm SD unless specified otherwise. $\mathrm{MVC}=$ Motor vehicle crash, $\mathrm{RVC}=$ recreation vehicle crash; $\mathrm{AA}=\mathrm{African} \mathrm{American} .{ }^{*}$ Performed by $\chi^{2}$ test.

and the absence of concurrent head injury was confirmed by medical records and/or physician reports of a relevant history, physical examination findings and, when available, clinical imaging results (i.e. negative $\mathrm{CT}$ ). $\mathrm{OI}$ in this cohort primarily resulted from sports- and play-related injuries and falls. All but 4 controls were right-hand dominant. Detailed information on the demographic and injury characteristics of both groups is presented in table 1 .

Potential participants in both groups were excluded if there was evidence of previous head injury, child abuse, preexisting neurologic disorders (e.g. cerebral palsy, mental retardation and epilepsy) or diagnosed learning disabilities, preexisting severe psychiatric disorders (e.g. schizophrenia or pervasive developmental disorders), prematurity or low birth weight (birth weight of $<2,500 \mathrm{~g}$ and gestation of $<37$ weeks), penetrating gunshot wounds to the brain, contraindication to undergoing MRI, hypoxia $\left(\mathrm{PO}_{2}<96 \mathrm{~mm} \mathrm{Hg}\right.$ for age of 6-16 years) or hypotension (systolic blood pressure $2 \mathrm{SD}$ below the mean for the age group).

\section{Imaging}

All participants underwent MRI without sedation on Philips 1.5-Tesla Intera scanners [Netsch et al., 2001] at the Texas Children's Hospital (Houston), the Rogers MRI Center, the University of Texas Southwestern Medical Center (Dallas), or the Miami Children's Hospital (Miami), using comparable platforms and software. Regular quality assurance testing was performed on all 3 scanners, including American College of Radiology phantom and Weisskoff testing for echo-planar imaging sequences, and all scanners were consistently noted to be within an acceptable range throughout the study.

DTI Acquisition. Transverse multislice spin echo, single-shot, echo-planar imaging sequences $(10,150.5 \mathrm{~ms}$ repetition time; 90 $\mathrm{ms}$ echo time; $2.7-\mathrm{mm}$-thick slices with 0 -mm gap) were applied. A $256-\mathrm{mm}$ field of view (FOV; receiver $\mathrm{FOV}=100 \%$ ) was used with a measured voxel size of $2.69 \times 2.69 \times 2.7 \mathrm{~mm}$. Diffusivities were evaluated along 15 directions (number of $\mathrm{b}$ value $=2$; low $\mathrm{b}$ value $=0 \mathrm{~s} / \mathrm{mm}^{2}$; high $b$ value $=860 \mathrm{~s} / \mathrm{mm}^{2}$ ). Two acquisitions of high-b images were obtained and averaged to ensure a better signal-to-noise ratio. A total number of 55 slices were acquired, and each acquisition took approximately $6 \mathrm{~min}$.

Volumetric Acquisition. $\mathrm{T}_{1}$-weighted 3-D sagittal acquisition series were used for volumetric analysis. The parameters included $15 \mathrm{~ms}$ repetition time, $4.6 \mathrm{~ms}$ echo time, $1.0-\mathrm{mm}$-thick slices with 0 -mm gap. A $256-\mathrm{mm}$ FOV (receiver FOV $=100 \%$ ) was used with a voxel size of $1.0 \times 1.0 \times 1.0 \mathrm{~mm}$.

DTI Analysis. Prior to computing FA maps with the Philips fiber tracking 4.1v3 Beta 2 software, shear and eddy current distortion as well as head motion artifact were corrected by using the Philips Pride registration tool [Netsch et al., 2001]. Regions of interest of the CC were manually traced on the midsagittal plane, following a previously published protocol [Wilde et al., 2006], and included the genu/anterior, body/central and splenium/posterior subregions as well as the total CC. The automated Philips 3-D fiber tracking program was utilized to examine fiber tracks passing through the selected regions in the CC. Mean FA and mean ADC were used as quantitative DTI variables. The algorithm for fiber tracking is based upon the fiber assignment by continuous tracking method [Mori et al., 1999]. If the FA in the voxels was $<0.2$, or if the angle between adjacent voxels was $>7^{\circ}$, fiber tracking terminated.

Intra- and Interrater Reliability. DTI analysis was performed by 2 experienced raters, following the specified protocol. To ensure intrarater reliability, each region of interest was independently analyzed by each rater twice. To examine interrater agreement, 2 trained raters performed an analysis of each subregion in a sample of 6 participants randomly selected ( 3 TBI patients and 3 controls). Shrout-Fleiss intraclass correlation coefficients were 
calculated and revealed satisfactory intra- (range: 0.937-1.000) and interrater reliability (range: 0.913-0.976).

Volumetric Analysis. Volumetric segmentation was performed by the Freesurfer image analysis suite version 4.5.0 (Athinoula A. Martinos Center for Biomedical Imaging, 2005; http://surfer. nmr.mgh.harvard.edu/). In order to reduce bias with respect to a particular time point, the longitudinal processing scheme was initialized with a template based on the cross-sectionally processed scans at both 3 and 18 months. Details of the cross-sectional [Fischl et al., 2004] and longitudinal [Morey et al., 2010] procedures have been described previously. Briefly, cross-sectional processing involves intensity normalization [Sled et al., 1998], automated Talairach transformation, the removal of non-brain tissue using a hybrid watershed/surface deformation procedure, and segmentation and labeling of the subcortical white matter and deep gray matter volumetric structures [Fischl et al., 2002, 2004]. Each scan was aligned to the unbiased template space, and the segmentation of the template was used as an initial approximation for the segmentation of each time point in longitudinal processing. The use of the unbiased template for initializing the processing of cases in a longitudinal series is intended to reduce the random variation in the longitudinal procedure and improve the robustness and sensitivity of the overall longitudinal analysis, as stated by the Freesurfer developers. Results for each subject were visually inspected to ensure accuracy of registration, skull stripping and segmentation. No editing was necessary in the automated segmentation of subcortical structures. The 5 standard subregions of the CC generated by Freesurfer were used in the analysis (anterior, midanterior, central, midposterior and posterior), as well as a composite for the total CC, which was the sum of the volumes of each of these subregions. The CC volumes derived from Freesurfer are considered true volume estimates that compose the entire structure, with partial volume correction in order to account for the fraction of the structure in each border voxel. The anterior, central and posterior subregions used in volumetric analysis corresponded to the genu/anterior, body/central and splenium/posterior subregions, respectively, that were used in DTI analysis.

\section{Cognitive Measure}

The baseline condition of the arrow flanker test [Bunge et al., 2002; Eriksen and Eriksen, 1974] was selected as a measure of information processing speed because we found that this measure of visual choice reaction time (RT; without distraction) was related to CC integrity as measured by DTI variables [Levin et al., 2008; Wilde et al., 2006]. One advantage of using this particular cognitive measure is that it is a language-free and multitrial task with a high reliability (split-half $r=0.96$ ) in the TBI population. Missing data were due to technical problems with this computerized task, the child's refusal to complete the task, or time constraints of the parent/guardian which precluded administration. There did not appear to be any source of systematic bias for the subjects with missing data, such as greater injury severity (i.e. GCS score) or cognitive impairment, and data were missing from all 3 sites. In the baseline condition of this task, a horizontal central arrow pointing to the left or to the right appeared on each trial. The child was asked to quickly press the button on the right or the left consistent with the direction that the arrow was pointing in. There were 28 trials in the baseline task condition. The RT in each condition was the performance measure.

Longitudinal Changes in CC following Pediatric TBI

\section{Data Analysis}

Demographic statistics between groups were compared using $t$ tests for continuous variables, i.e. (1) age at injury, (2) socioeconomic composite index (SCI) z-score, a measure of socioeconomic status [Yeates et al., 1997], (3) maternal education, and (4) time after injury for both the 3-and 18-month assessment time points. Categorical variables were analyzed using the $\chi^{2}$ (for gender) or Fisher's exact test (for handedness, race/ethnicity distribution and mechanism of injury). Moreover, $t$ tests were used to examine the group differences in FA and ADC of the CC subregions and total CC at both 3 and 18 months. The group differences in CC subregion and total CC volumes at 3 and 18 months were also analyzed by general linear models, including total intracranial volume as a covariate to account for differences in head size. To examine the within-group changes over time in FA, ADC and volumes of the CC subregions and total $\mathrm{CC}$, paired $\mathrm{t}$ tests were used for each group and each subregion on the difference score, as calculated by the 3 -month minus 18 -month values. A two-sample $t$ test was used to test the group difference on the change (twoway interaction of group by occasion). To examine the possibility that a complicated mild injury may differ in degree from more severe injuries on DTI, we repeated the analyses excluding the participants with complicated mild TBI and found that this did not change the results of the analyses. The within-group differences on the flanker baseline RT between 3 and 18 months were tested using a paired t test of the difference score between RT at 3 and 18 months. Pearson's partial correlation coefficients with age at injury and SCI controlled for were used to examine the relation of DTI and volumetric variables to performance on flanker baseline RT at both 3 and 18 months. The significance level was adjusted by using the family-wise Bonferroni method for the subregions ( 3 subregions for DTI, $\alpha=0.017 ; 5$ subregions for volumetrics, $\alpha=0.01)$.

\section{Results}

\section{Demographic and Injury Characteristics}

The TBI group did not significantly differ from the OI group in age, gender, handedness, SCI, maternal education or the duration of the postinjury interval at either the 3- or 18-month assessment point. As expected, groups differed significantly in the mechanism of injury, with more participants in the TBI group sustaining injury via motor vehicle and motorcycle crashes, and more participants in the OI group sustaining injury via sports- and play-related injuries and falls. $t$ values (or $\chi^{2}$ or Fisher's exact test values) and $\mathrm{p}$ values are presented in table 1.

\section{Group Differences at 3 and 18 Months after Injury for DTI}

At 3 months after injury, the FA of the genu/anterior, body/central, splenium/posterior and total CC differed significantly between the groups, with the TBI group demonstrating decreased FA. Similarly, the ADC for the 
Table 2. FA group difference for CC at 3 and 18 months

\begin{tabular}{|c|c|c|c|c|c|}
\hline Regions & $\begin{array}{l}\text { Interval } \\
\text { months }\end{array}$ & TBI & OI & $\mathrm{t}$ & $\mathrm{p}$ \\
\hline \multirow[t]{2}{*}{ Total CC } & 3 & $0.42 \pm 0.03$ & $0.47 \pm 0.02$ & 5.03 & $<0.0001$ \\
\hline & 18 & $0.43 \pm 0.03$ & $0.47 \pm 0.02$ & 2.36 & 0.043 \\
\hline \multirow[t]{2}{*}{ Genu } & 3 & $0.42 \pm 0.03$ & $0.47 \pm 0.03$ & 5.37 & $<0.0001$ \\
\hline & 18 & $0.43 \pm 0.03$ & $0.48 \pm 0.02$ & 6.97 & $<0.0001$ \\
\hline \multirow[t]{2}{*}{ Body } & 3 & $0.41 \pm 0.04$ & $0.45 \pm 0.03$ & 4.16 & 0.0001 \\
\hline & 18 & $0.42 \pm 0.03$ & $0.46 \pm 0.02$ & 2.36 & 0.043 \\
\hline \multirow[t]{2}{*}{ Splenium } & 3 & $0.46 \pm 0.04$ & $0.49 \pm 0.02$ & 4.70 & 0.0004 \\
\hline & 18 & $0.47 \pm 0.04$ & $0.51 \pm 0.02$ & 3.64 & 0.003 \\
\hline
\end{tabular}

Italics indicate significance after Bonferroni correction for multiple comparisons.

genu/anterior, splenium/posterior and total CC differed significantly between the groups, with the TBI group demonstrating increased ADC. The ADC for the body/ central CC subregion exhibited a positive trend, but this was not considered significant after correction for multiple comparisons.

At the 18-month postinjury assessment, the FA for the genu/anterior and splenium/posterior subregions, and the ADC for all subregions and total CC differed significantly between the groups, with lower FA and higher ADC in the TBI group. FA for the body/central subregion and total CC demonstrated a trend after Bonferroni correction. Tables 2 and 3 detail means, $\mathrm{SD}, \mathrm{t}$ and $\mathrm{p}$ for all $\mathrm{CC}$ regions at both time points.

\section{Longitudinal DTI Analyses}

Both groups demonstrated increased FA in total CC and all CC subregions at 18 months as compared to 3 months after injury. However, only the increase in FA in the splenium/posterior subregion in both the TBI $[\mathrm{t}(22)=-3.36 ; \mathrm{p}=0.003]$ and $\mathrm{OI}[\mathrm{t}(24)=-2.76 ; \mathrm{p}=0.011]$ groups reached significance after correction for multiple comparisons. The TBI group showed a marginally significant increase in FA for the total CC $[\mathrm{t}(22)=-2.21 ; \mathrm{p}=$ 0.038 ] over time. The OI group showed a marginally significant increase in FA in the total CC [t(24) = -2.04; $\mathrm{p}=$ $0.053]$ and marginally significant decreases in ADC for the body/central subregion $[\mathrm{t}(24)=2.10 ; \mathrm{p}=0.047]$ and total CC $[\mathrm{t}(24)=1.76 ; \mathrm{p}=0.092]$. No significant betweengroup differences for longitudinal changes were noted in any region. Figure 1 illustrates the change between 3 and
Table 3. ADC group difference for CC at 3 and 18 months

\begin{tabular}{lclllc}
\hline Regions & $\begin{array}{l}\text { Interval } \\
\text { months }\end{array}$ & $\begin{array}{l}\text { TBI } \\
10^{-3} \mathrm{~mm}^{2} / \mathrm{s}\end{array}$ & $\begin{array}{l}\text { OI } \\
10^{-3} \mathrm{~mm}^{2} / \mathrm{s}\end{array}$ & $\mathrm{t}$ & $\mathrm{p}$ \\
\hline Total CC & 3 & $0.98 \pm 0.07$ & $0.93 \pm 0.05$ & -3.03 & 0.004 \\
& 18 & $0.99 \pm 0.07$ & $0.91 \pm 0.06$ & -4.30 & $<0.0001$ \\
\hline Genu & 3 & $0.91 \pm 0.05$ & $0.86 \pm 0.04$ & -3.80 & 0.0004 \\
& 18 & $0.93 \pm 0.07$ & $0.85 \pm 0.05$ & -4.58 & $<0.0001$ \\
\hline Body & 3 & $0.97 \pm 0.10$ & $0.92 \pm 0.07$ & -2.06 & 0.046 \\
& 18 & $0.97 \pm 0.08$ & $0.90 \pm 0.06$ & -3.43 & 0.001 \\
\hline Splenium & 3 & $1.02 \pm 0.09$ & $0.95 \pm 0.06$ & -3.18 & 0.003 \\
& 18 & $1.04 \pm 0.10$ & $0.95 \pm 0.07$ & -3.97 & 0.0003 \\
& & & & &
\end{tabular}

ADC values are means \pm SD. Italics denote significance after Bonferroni correction for multiple comparisons.
18 months for FA and ADC of both the splenium/posterior subregion of the CC and the total CC in both groups.

\section{Relation of DTI to Cognitive Functioning}

Cognitively, the OI group demonstrated a significant improvement in speed of information processing over time $[\mathrm{t}(18)=2.55 ; \mathrm{p}=0.02]$. The speed of information processing did not differentiate the TBI and OI groups at either the 3- or 18-month occasion when all participants were included. However, when the participants with complicated mild injuries were excluded, the results were unchanged at 3 months, but there was a trend for group difference at 18 months $[\mathrm{t}(28)=-1.9 ; \mathrm{p}=0.086 ; \mathrm{d}=1.25]$ such that the participants with TBI had slower RT.

At 3 months after injury in the TBI group, FA in the splenium $(\rho=-0.58 ; p=0.008)$ and total CC $(\rho=-0.55$; $\mathrm{p}=0.012$ ) were significantly correlated with baseline condition processing speed. Higher FA was related to faster RT. These correlations became stronger at the 18-month postinjury occasion (for the splenium/posterior subregion: $\rho=-0.84, p=0.001$; for total CC: $\rho=-0.71, p=$ $0.014)$. Figure 2 reflects the relation between FA in the splenium/posterior subregion of the CC and RT on the flanker task in each group.

\section{Group Differences at 3 and 18 Months after Injury for Volumetrics}

At 3 months after injury, the midanterior $(\mathrm{F}(1,37)=$ $6.25, \mathrm{p}=0.017)$ and body/central $[\mathrm{F}(1,37)=5.36 ; \mathrm{p}=$ $0.026]$ subregions and total $\mathrm{CC}[\mathrm{F}(1,37)=4.27 ; \mathrm{p}=0.046]$ marginally differed between the groups though the genu/ 

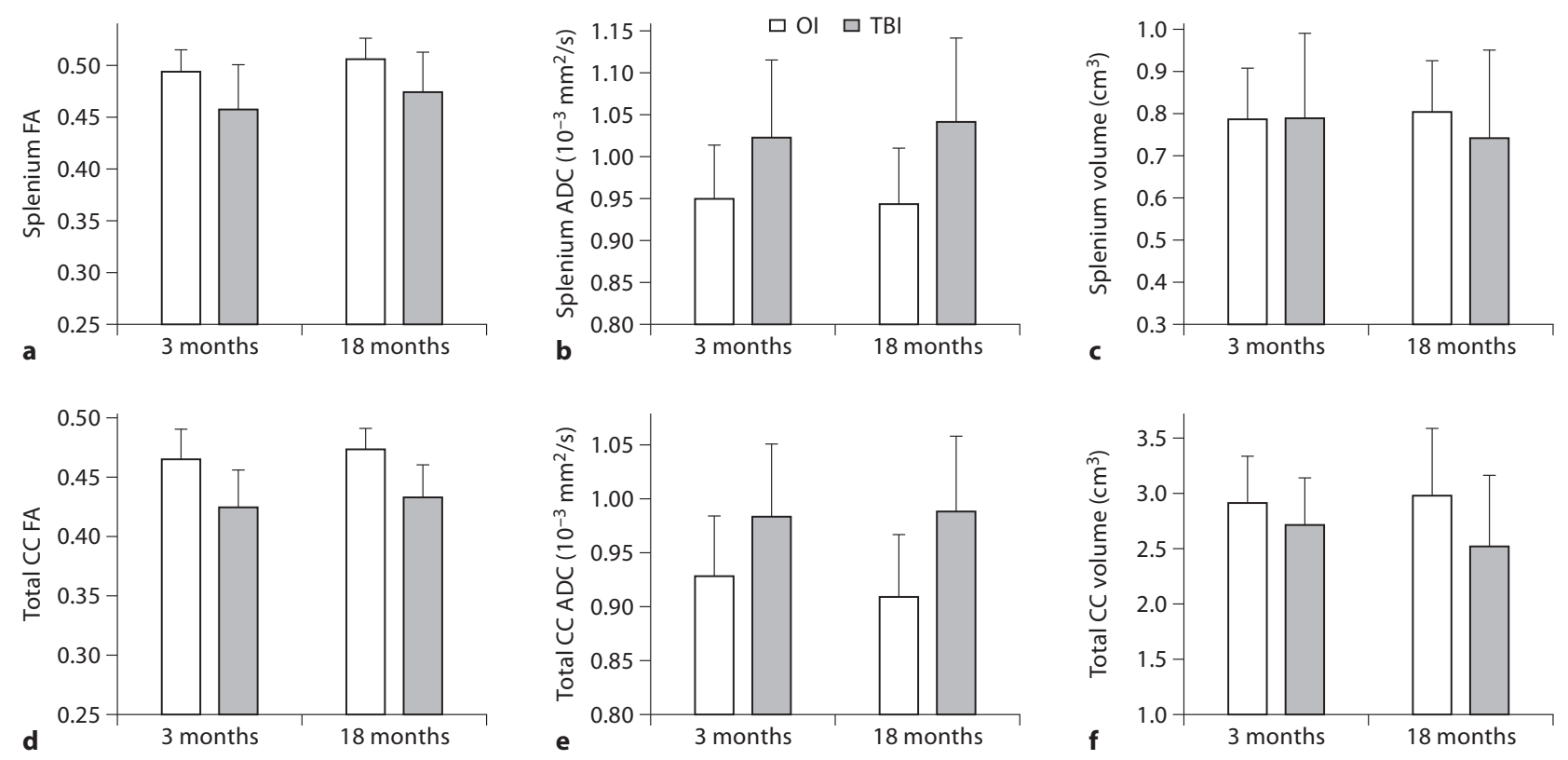

Fig. 1. Bar graphs with error bars. Pattern of longitudinal change between 3 and 18 months after injury in FA (a) and ADC (b) as measured by quantitative DTI tractography, and volume (c) as measured by volumetric analysis in the splenium/posterior subregion of the $\mathrm{CC}$ in both the TBI and OI groups. Note that the FA remains significantly lower in the TBI group as compared to the OI group, but increases comparably to the OI group. The ADC decreases over time in the OI group, as would be expected given normal developmental change, but the ADC in the TBI group continues to increase at a modest rate, which is unexpected given the typical relation between FA and ADC. The volume of the splenium increases in the OI group, as expected given normal developmental change that is occurring in this age range, but evidences continued degenerative change in the TBI group over time. Changes in total CC are reflected in FA (d) and ADC (e) as measured by quantitative DTI tractography, and in volume (f) as measured by volumetric analysis.

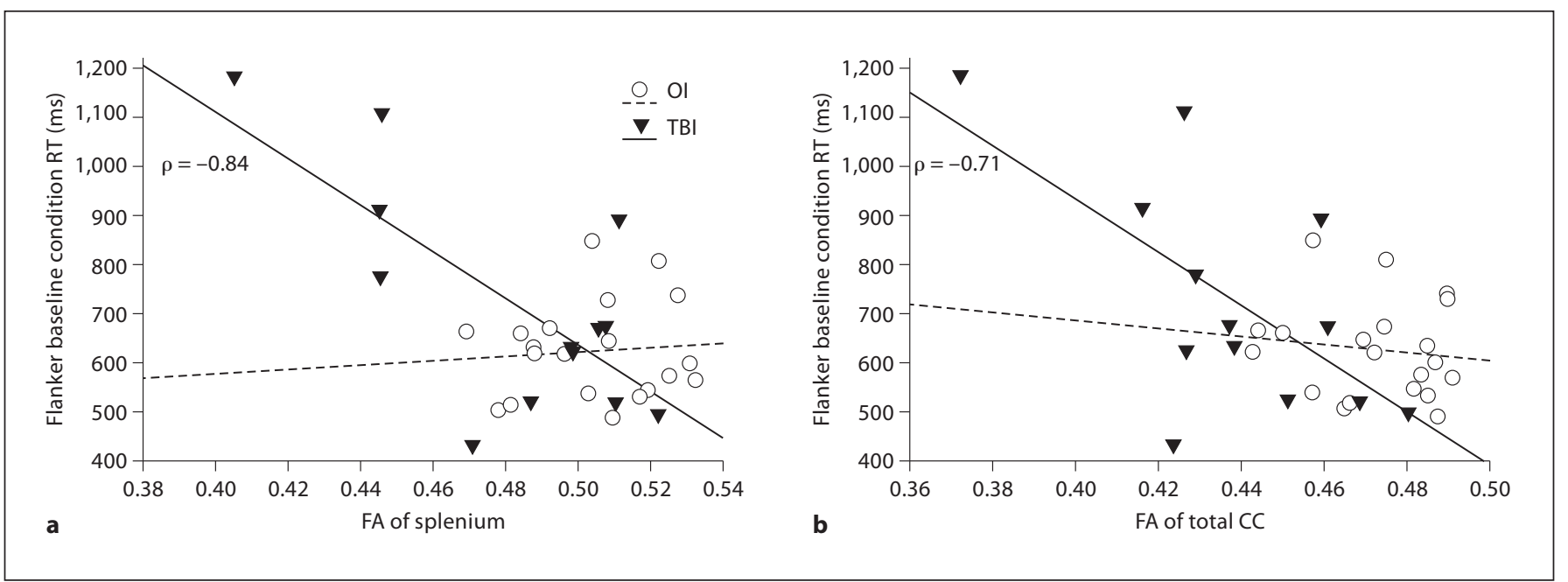

Fig. 2. Scatter plots with regression lines demonstrating the relation between flanker baseline condition RT and FA of the splenium/posterior subregion (a) and of the total CC (b) at 18 months after injury in the TBI and OI groups. Note the significant correlation for the TBI group. 
Table 4. Volumetric differences in CC subregions at 3 and 18 months

\begin{tabular}{lrllrl}
\hline Regions & $\begin{array}{r}\text { Interval } \\
\text { months }\end{array}$ & $\begin{array}{l}\mathrm{TBI}^{1} \\
\mathrm{~cm}^{3}\end{array}$ & $\begin{array}{l}\mathrm{OI}^{1} \\
\mathrm{~cm}^{3}\end{array}$ & \multicolumn{2}{l}{$\mathrm{F}$} \\
\hline Total CC & 3 & 2.72 & 2.92 & 4.27 & 0.046 \\
& 18 & 2.52 & 2.99 & 11.83 & 0.002 \\
\hline Anterior & 3 & 0.72 & 0.79 & 3.19 & 0.082 \\
& 18 & 0.67 & 0.80 & 7.76 & 0.008 \\
\hline Midanterior & 3 & 0.41 & 0.46 & 6.25 & 0.017 \\
& 18 & 0.37 & 0.47 & 15.13 & 0.0004 \\
\hline Central & 3 & 0.40 & 0.47 & 5.36 & 0.026 \\
& 18 & 0.38 & 0.48 & 10.20 & 0.003 \\
\hline Midposterior & 3 & 0.40 & 0.42 & 1.57 & 0.218 \\
& 18 & 0.36 & 0.44 & 8.96 & 0.005 \\
\hline Posterior & 3 & 0.79 & 0.79 & 0.11 & 0.740 \\
& 18 & 0.74 & 0.81 & 2.14 & 0.152 \\
\hline
\end{tabular}

Italics indicate significance after Bonferroni correction for multiple comparisons.

anterior and splenium/posterior regions did not significantly differ (table 4). In each case, the TBI group exhibited a decreased volume in the subregion relative to the OI comparison group. At 18 months after injury, the genu/anterior $[\mathrm{F}(1,37)=7.76 ; \mathrm{p}=0.008]$, midanterior $[\mathrm{F}(1$, $37)=15.13 ; \mathrm{p}=0.0004]$, body/central $[\mathrm{F}(1,37)=10.20$; $\mathrm{p}=0.003]$ and midposterior $[\mathrm{F}(1,37)=8.96 ; \mathrm{p}=0.005]$ subregions of the CC as well as the total $\mathrm{CC}[\mathrm{F}(1,37)=$ $11.83 ; \mathrm{p}=0.002$ ] significantly differed between the groups (see table 4 for least squares' means), again with the TBI group demonstrating significantly decreased volume relative to the OI group.

\section{Longitudinal Volumetric Analyses}

In terms of within-group differences, in the OI group, the genu/anterior $(\mathrm{p}=0.010)$, midposterior $(\mathrm{p}=0.002)$ and splenium/posterior $(\mathrm{p}=0.0008)$ subregions of the CC as well as the total CC $(\mathrm{p}=0.001)$ demonstrated significant volume increases between 3 and 18 months after injury. The midanterior and body/central subregions marginally differed, again demonstrating an increase in volume over time, but this change was not significant. In contrast, the TBI group demonstrated decreases in volume between 3 and 18 months after injury in the genu/ anterior $(\mathrm{p}=0.009)$ and midposterior $(\mathrm{p}=0.007)$ subregions and total CC $(\mathrm{p}=0.012)$. Change in $\mathrm{CC}$ volume be- tween 3 and 18 months revealed significant betweengroup differences in the genu/anterior [Satterthwaite $\mathrm{t}(17.8)=-3.70 ; \mathrm{p}=0.002$ ], midanterior [Satterthwaite $\mathrm{t}(20)=-2.95 ; \mathrm{p}=0.008$ ], midposterior [Satterthwaite $\mathrm{t}(20)=-4.19 ; \mathrm{p}=0.0004]$ and splenium/posterior [Satterthwaite $\mathrm{t}(16.5)=-3.20 ; \mathrm{p}=0.005]$ regions and the total CC [Satterthwaite $\mathrm{t}(17.2)=-3.73 ; \mathrm{p}=0.002]$. A marginally significant difference was noted for the body/central subregion [t(38) $=-1.82 ; \mathrm{p}=0.077]$. Figure $1 \mathrm{c}$ and $2 \mathrm{c}$ illustrate the pattern of change between 3 and 18 months in the TBI and OI groups for the splenium/posterior subregion and the total $\mathrm{CC}$, respectively.

\section{Relation of Volume to Cognitive Functioning}

There were no significant correlations between processing speed and volumes of the subregions of the CC in either group at 3 and 18 months after injury interval assessments. Total CC volume was marginally related to processing speed at 18 months in the OI group only ( $\rho=$ $-0.433 ; p=0.06$ ), with greater volume associated with faster processing speed.

\section{Discussion}

This study demonstrated continued late degenerative change as reflected in the gross morphometry of nearly all regions of the CC, as measured by volumetric analysis between 3 and 18 months after injury in the TBI group. These degenerative changes were most prominent in the genu/anterior, midposterior and splenium/posterior subregions of the CC. In contrast, the genu/anterior, midposterior and splenium/posterior subregions of the CC demonstrated significant volume increases in the comparison group of children with OI, consistent with known developmental changes in this age range [Löbel et al., 2009; Zhang et al., 2005]. Previous developmental studies have demonstrated that the thinly myelinated and densely packed fibers more characteristic of the genu mature later than those with thicker myelination [Kochunov et al., 2010], and hence the developmental changes may be greater in these regions during the age range of the children whom we studied. It is also possible that these fibers may demonstrate a differential vulnerability to aging [Kochunov et al., 2010] or insult, including TBI-induced axonal injury in particular [Reeves et al., 2005]. Traumainduced atrophy of the CC likely occurs as a consequence of complex neuropathological features that involve both direct trauma to the CC [MacDonald et al., 2007b; Parizel et al., 1998] as well as secondary degeneration of a Wal- 


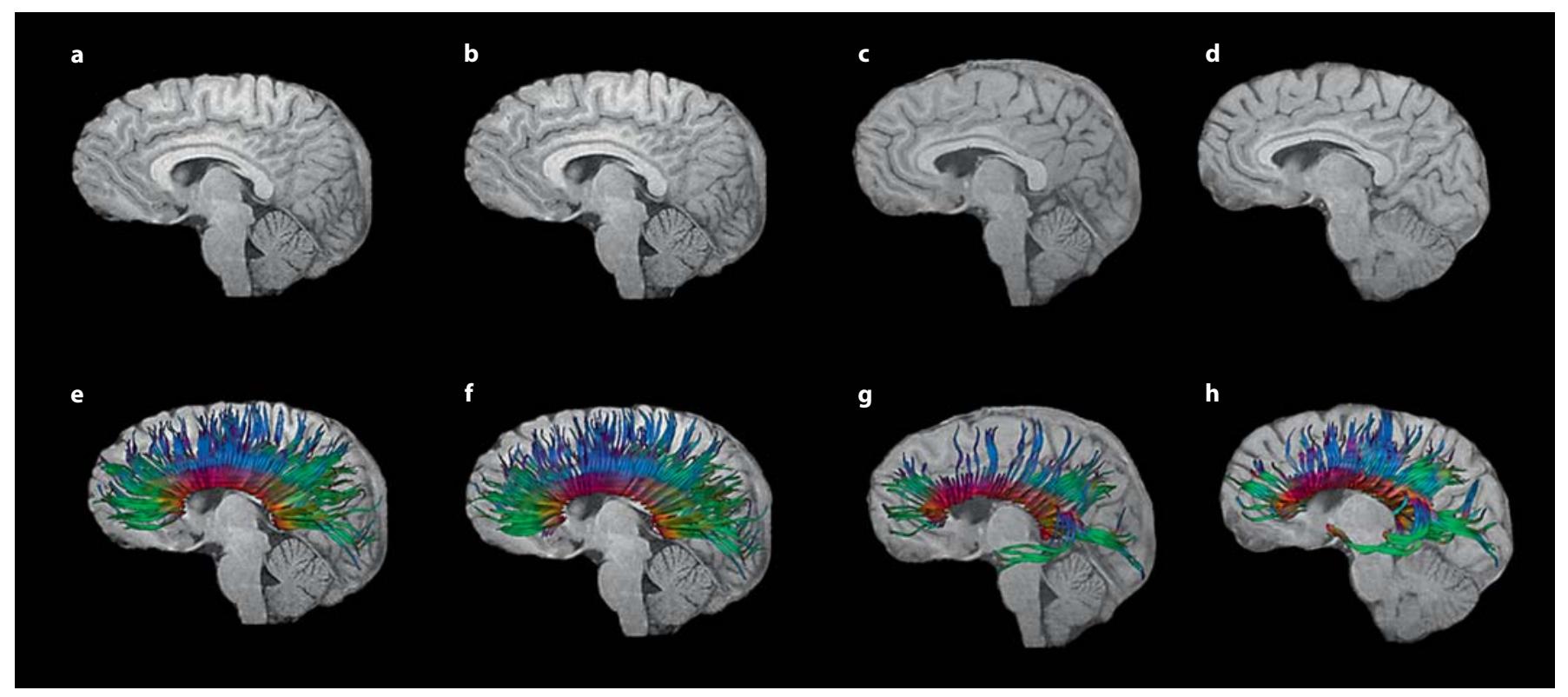

Fig. 3. $\mathrm{T}_{1}$-weighted sagittal images of a 16-year-old male adolescent with OI at 3 months (a) and 18 months (b) after injury. $\mathrm{T}_{1^{-}}$ weighted images of a 15-year-old male adolescent with moderate TBI (GCS score of 9) at 3 months (c) and 18 months (d) after injury. Note the subtle atrophy of the CC in the TBI child. DTI tractography of the CC is overlaid on the $\mathrm{T}_{1}$-weighted image for the adolescent with OI at 3 months (e) and 18 months (f) after injury,

lerian type occurring because diffuse damage elsewhere in the brain disrupts the integrity of white matter tracts [Di Paola et al., 2010].

Typical CC development in childhood follows a predictable course of myelination, which, at least in animal models, also relates to environmental complexity [Markham et al., 2009]. In the typically developing brain, this likely also leads to complex brain-environment-developmental interactions resulting in increased CC volume during the age ranges of the children in this study and as confirmed by the results of the OI participants. However, brain injury may induce deficits in cognitive processing which likely diminish the child's ability to process complex material [Taylor, 2004]. Hampered by slowed processing speed and diminished integration of interhemispheric information due to the CC damage, this could theoretically interact negatively with development and minimize CC growth trajectories. As reflected in further reduced volume over time, the TBI children did not exhibit maturation-related volume increases in the CC. Likewise, it is not unexpected that processing speed would improve in typically developing children, as was observed in the children with OI, but not in TBI partici- which reflects a subtle qualitative increase in fiber density. Similarly, the DTI tractography of the CC overlaid on the $\mathrm{T}_{1}$-weighted image from the adolescent with TBI at 3 months (g) and 18 months (h) after injury reveals a qualitative increase in both fiber density and intensity of the CC over time though the density of fibers is still significantly lower than that of the comparison OI participant.

pants. These findings support the notion that brain injury disrupts normal white matter maturation and development, disconnecting the typical link with maturational changes that improve processing speed.

However, the current study shows a most interesting, apparent adaptation in CC functioning in that children with TBI could adequately perform the flanker task, at a level that did not differ from that of the OI controls at the 3-month baseline or 18-month follow-up. This suggests that within the initial 3-month postinjury time frame, adaptations in CC functioning were sufficient for these patients to adequately perform the task despite the volume and DTI changes that had already occurred at that stage. In case of surgical treatment of the CC, typically performed as a procedure to treat chronic epilepsy, adaptive rerouting of CC pathways has long been documented [Bloom and Hynd, 2005; Tanriverdi et al., 2009]. This is likely part of the explanation for the TBI group that part of the recovery from TBI is some form of programmed adaptive mechanism that engages intact tracts to adapt. It is also possible that new inter- and intrahemispheric communications could potentially be formulated via alternative routes such as the anterior commissure [Patel et 
al., 2010]. Even though the TBI group was not significantly slower than the OI group on the flanker task, DTI findings did relate to processing speed in the TBI group in the splenium/posterior subregion, which became stronger in this group over time.

The continued degenerative changes over this time interval on volumetrics in the TBI group were not reflected in longitudinal changes in DTI metrics such as FA. Despite loss of volume in the TBI group in some subregions, likely reflective of some degree of neuronal death and axonal degeneration, FA generally increased in both the TBI and the OI groups in all regions. In animal studies, histological findings have related tissue volume loss to cellular loss [Grady et al., 2003] and, specifically, CC volume loss to neuronal loss [Bramlett and Dietrich, 2002]. On the other hand, DTI is likely showing how injured neurons, while damaged, recover over time [Kumar et al., 2010], at least to some degree. In the current study, these two methods likely reflect quite different neuropathological endpoints over time where reduced volume reflects overall neuronal loss, whereas DTI findings more specifically reflect axonal integrity and restorative and adaptive processes that develop over time after injury. Figure 3 illustrates the qualitative differences in DTI tractography over time that complement the quantitative findings we describe in this study in one participant. However, it should be noted that although FA in the TBI group did increase, the ADC in the TBI group did not decrease, as would have been anticipated given the traditional inverse relation between FA and $\mathrm{ADC}$, and in fact, the ADC also increased over time. This suggests that the expected developmental trajectory reflected in FA increase and ADC increase observed in the comparison group may, in fact, be altered somewhat by TBI despite the apparent increase in FA. As both FA and ADC may be impacted by multiple factors related to the microstructural characteristics of the tissue, including fiber density, axonal diameter and the ratio of intracellular/ extracellular space, further investigation into the more specific nature of these changes is warranted.

Although the trajectory of change in FA for both groups followed a similar slope after injury, the difference between the TBI and OI groups remained significant, with the TBI group exhibiting a significantly lower mean FA at both time points in several regions. This also reflects that DTI changes such as FA may be more specific to adaptive mechanisms of recovery than are traditional volumetric measures [van der Zijden et al., 2008]. This difference further indicates that, neuropathologically, DTI is assessing something quite different than just neuronal loss or axonal degradation. Because DTI related to processing speed, it may be the more sensitive biomarker of actual CC integrity than measuring atrophic changes via volume loss. Because DTI is such a new technology, studies are just now being done to ascertain whether microstructural changes as found by DTI may occur independently of volumetric parenchymal changes [Canu et al., 2010]. Clearly, multimodality imaging studies may be useful in any further examination of TBI-related consequences to brain parenchyma.

\section{Strengths, Limitations and Future Directions}

Only few studies have specifically examined longitudinal changes following TBI using advanced imaging modalities such as volumetric analysis and DTI [Bendlin et al., 2008; Kumar et al., 2009, 2010; Sidaros et al., 2008, 2009], and to our knowledge, no study has examined these changes by DTI in a pediatric TBI population [Levin et al., 2000]. Strengths of this study include its prospective, longitudinal design and its use of an orthopedically injured comparison group.

We limited this investigation to changes in the CC, but additional brain regions should be examined in future studies. As we have shown elsewhere, diffuse changes in the brain result from moderate-to-severe TBI [Bigler et al., 2010], including thinning of the cerebral cortex [Merkley et al., 2008], but how these diffuse changes relate specifically to the atrophic changes in the CC and cognitive outcome is not known at this time. Additionally, other methods of DTI analysis such as voxel-based analysis and region-of-interest analysis will be applied in future studies to examine these differences.

We emphasize that these analyses represent group trends, and we acknowledge the possibility that different patterns of change over time may emerge in some individuals with TBI based upon factors related to the heterogeneity of injury type and location, age at injury, mechanism of injury, and pre- and postinjury characteristics that may render some individuals more resilient to the effects of injury than others. Future studies may examine some of these factors.

Finally, missing data may have limited the power to detect differences in the behavioral and cognitive measures. For instance, the effect size for group differences in flanker task RT at 3 months was 0.37 , which is small to moderate, but the power to detect a significant difference was only $33.7 \%$. Similarly at 18 months, the effect size was 0.62 , which is moderate to large, but the power to detect a significant difference was only $43.4 \%$. 


\section{Conclusions}

The current findings indicate that at the macro- and microstructural levels, two seemingly opposite trajectories were noted following moderate-to-severe TBI in children and adolescents. Volumetric analyses demonstrated degeneration in the CC over time, whereas DTI findings showed some degree of increase in FA over time, indicative of maturation or recovery. FA was also related to speed of information processing at both time points though even more strongly at the 18-month postinjury interval. White matter changes as measured by multimodality imaging studies may be useful for better understanding changes indicative of both degeneration and residual loss of tissue and cognitive difficulty as well as plasticity and recovery, particularly in an age range that is also undergoing dynamic developmental change.

\section{Acknowledgments}

This research was supported by grant NS-21889 awarded to H.S.L. by the National Institutes of Health. We would also like to acknowledge the generous support by Mission Connect of the TIRR Foundation. We acknowledge the contribution of Stacey K. Martin by assisting in manuscript preparation, and the technical assistance of Tracy J. Abildskov. We also wish to thank Ms. Lori Cook and Drs. Sandra B. Chapman and Gillian Hotz. We would also like to thank the patients and their families for involvement in this study. This research paper is also the work of a doctoral dissertation.

\section{References}

Alexander AL, Lee JE, Lazar M, Field AS (2007): Diffusion tensor imaging of the brain. Neurotherapeutics 4:316-329.

-Anderson CV, Bigler ED (1994): The role of caudate nucleus and corpus callosum atrophy in trauma-induced anterior horn dilation. Brain Injury 8:565-569.

Arfanakis K, Haughton VM, Carew JD, Rogers BP, Dempsey RJ, Meyerand ME (2002): Diffusion tensor MR imaging in diffuse axonal injury. Am J Neuroradiol 23:794-802.

-Ashwal S, Holshouser BA, Tong KA (2006): Use of advanced neuroimaging techniques in the evaluation of pediatric traumatic brain injury. Dev Neurosci 28:309-326.

-Assaf Y, Beit-Yannai E, Shohami E, Berman E, Chohen Y (1997): Diffusion- and $\mathrm{T}_{2}$-weighted MRI of closed-head injury in rats: a time course study and correlation with histology. Magn Reson Imaging 15:77-85.

-Aukema EJ, Caan MWA, Oudhuis N, Majoie CBLM, Vos FM, Reneman L, et al (2009): White matter fractional anisotropy correlates with speed of processing and motor speed in young childhood cancer survivors. Int J Radiat Oncol Biol Phys 74:837-843.

- Babikian T, Marion SD, Copeland S, Alger JR, O'Neill J, Cazalis F, et al (2010): Metabolic levels in the corpus callosum and their structural and behavioral correlates after moderate-to-severe pediatric TBI. J Neurotrauma 27:473-481.

Battistone M, Woltz D, Clark E (2008): Processing speed deficits associated with traumatic brain injury: processing inefficiency of cautiousness? Appl Neuropsychol 15:69-78.

Bazarian JJ, Zhong J, Blyth B, Zhu T, Kavcic V, Peterson D (2007): Diffusion tensor imaging detects clinically important axonal damage after mild traumatic brain injury: a pilot study. J Neurotrauma 24:1447-1459.
Beauchamp MH, Anderson VA, Catroppa C, Bloom JS, Hynd GW (2005): The role of the corMaller JJ, Godfrey C, Rosenfeld JV, et al (2009): Implications of reduced callosal area for social skills after severe traumatic brain injury in children. J Neurotrauma 26:16451654.

Belanger HG, Vanderploeg RD, Curtiss G, Warden DL (2007): Recent neuroimaging techniques in mild traumatic brain injury. J Neuropsychiatry Clin Neurosci 19:5-20.

Benavidez DA, Fletcher JM, Hannay HJ, Bland ST, Caudle SE, Mendelsohn DB, et al (1999): Corpus callosum damage and interhemispheric transfer of information following closed head injury in children. Cortex 35: 315-336.

-Bendlin BB, Ries ML, Lazar M, Alexander AL, Dempsey RJ, Rowley HA, et al (2008): Longitudinal changes in patients with traumatic brain injury assessed with diffusion-tensor and volumetric imaging. Neuroimage 42: 503-514.

Benson RR, Meda SA, Vasudevan S, Kou Z, Govindarajan KA, Hanks RA, et al (2007): Global white matter analysis of diffusion tensor images is predictive of injury severity in traumatic brain injury. J Neurotrauma 24: 446-459.

Biegon A, Eberling JL, Richardson BC (1994): Human corpus callosum in aging and Alzheimer's disease: a magnetic resonance imaging study. Neurobiol Aging 15:393-397.

Bigler ED, Abildskov T, Wilde EA, McCauley SR, Li X, Merkley TL, et al (2010): Diffuse damage in pediatric traumatic brain injury: a comparison of automated versus operatorcontrolled quantification methods. Neuroimage 50:1017-1026. pus callosum in interhemispheric transfer of information: excitation or inhibition? Neuropsychol Rev 15:59-71.

Bramlett HM, Dietrich WD (2002): Quantitative structural changes in white and gray matter 1 year following traumatic brain injury in rats. Acta Neuropathol 103:607-614.

- Bunge SA, Dudukovic NM, Thomason ME, Vaidya CJ, Gabrieli JD (2002): Immature frontal lobe contributions to cognitive control in children: evidence from fMRI. Neuron 33:301-311.

Canu E, McLaren DG, Fitzgerald ME, Bendlin BB, Zoccatelli G, Alessandrini F, et al (2010): Microstructural diffusion changes are independent of macrostructural volume loss in moderate-to-severe Alzheimer's disease. J Alzheimers Dis 19:963-976.

Chepuri NB, Yen Y-F, Burdette JH, Li H, Moody DM, Maldjian JA (2002): Diffusion anisotropy in the corpus callosum. Am J Neuroradiol 23:803-808.

Chu Z, Wilde EA, Hunter JV, McCauley SR, Bigler ED, Troyanskaya M, et al (2010): Voxelbased analysis of diffusion tensor imaging in mild traumatic brain injury in adolescents. Am J Neuroradiol 31:340-346.

Di Paola M, Luders E, di lulio F, Cherubini A, Passaflume D, Thompson PM, et al (2010): Callosal atrophy in mild cognitive impairment and Alzheimer's disease: different effects in different stages. Neuroimage 49:141149.

-Ducreus D, Huynh I, Fillard P, Renoux J, PetitLacour MC, Marsot-Dupuch K, et al (2005): Brain MR diffusion tensor imaging and fibre tracking to differentiate between two diffuse axonal injuries. Neuroradiology 47:604608. 
Eriksen BA, Eriksen CW (1974): Effects of noise letters upon the identification of a target letter in a nonsearch task. Percept Psychophys 16:143-149.

-Ewing-Cobbs L, Hasan KM, Prasad MR, Kramer L, Bachevalier J (2006): Corpus callosum diffusion anisotropy correlates with neuropsychological outcomes in twins disconcordant for traumatic brain injury. Am J Neuroradiol 27:879-881.

Ewing-Cobbs L, Prasad MR, Swank P, Kramer L, Cox CS Jr, Fletcher JM, et al (2008): Arrested development and disrupted callosal microstructure following pediatric traumatic brain injury: relation to neurobehavioral outcomes. Neuroimage 42:1305-1315.

- Fischl B, Salat DH, Busa E, Albert M, Dieterich M, Haselgrove JC, et al (2002): Whole brain segmentation: automated labeling of neuroanatomical structures in the human brain. Neuron 33:341-355.

- Fischl B, Salat DH, van der Kouwe AJ, Makris N, Segonne F, Quinn BT, et al (2004): Sequenceindependent segmentation of magnetic resonance images. Neuroimage 23:S69-S84.

Gorrie C, Duflou J, Brown J, Gibson T, Waite PM (2001): Extent and distribution of vascular brain injury in pediatric road fatalities. J Neurotrauma 18:849-860.

Grady MS, Charleston JS, Maris D, Witgen BM, Lifshitz J (2003): Neuronal and glial cell number in the hippocampus after experimental traumatic brain injury: analysis by stereological estimation. J Neurotrauma 20: 929-941.

-Huisman TAGM, Schwamm LH, Schaefer PW, Koroshetz WJ, Shetty-Alva N, Ozsunar Y, et al (2004): Diffusion tensor imaging as potential biomarker of white matter injury in diffuse axonal injury. Am J Neuroradiol 25: 370-376.

-Inglese M, Makani S, Johnson G, Cohen B, Silver J, Gonen O, et al (2005): Diffuse axonal injury in mild traumatic brain injury: a diffusion tensor imaging study. J Neurosurg 103: 298-303.

Johansson B, Berglund P, Ronnback L (2009): Mental fatigue and impaired information processing after mild and moderate traumatic brain injury. Brain Injury 23:10271040.

Jokinen H, Ryberg C, Halska H (2007): Corpus callosum atrophy is associated with mental slowing and executive deficits in subjects with age-related white matter hyperintensities: the LADIS study. J Neurol Neurosurg Psychiatry 78:491-496.

- Kim J, Avants B, Patel S, Whyte J, Coslett BH, Pluta J, et al (2008): Structural consequences of diffuse traumatic brain injury: a large deformation tensor-based morphometry study. Neuroimage 2008:1014-1026.

Kochunov P, Williamson DE, Lancaster J, Fox P, Cornell J, Blangero J, et al (2010): Fractional anisotropy of water diffusion in cerebral white matter across the lifespan. Neurobiol Aging, E-pub ahead of print.
Kraus MF, Susmaras T, Caughlin BP, Walker CJ, Sweeney JA, Little DM (2007): White matter integrity and cognition in chronic traumatic brain injury: a diffusion tensor imaging study. Brain 130(pt 10):2508-2519.

Kumar R, Husain M, Gupta RK, Hasan KM, Haris M, Agarwal AK, et al (2009): Serial changes in the white matter diffusion tensor imaging metrics in moderate traumatic brain injury and correlation with neuro-cognitive function. J Neurotrauma 26:481-495.

- Kumar R, Saksena S, Husain M, Srivastava A, Rathore RK, Agarwal S, et al (2010): Serial changes in diffusion tensor imaging metrics of corpus callosum in moderate traumatic brain injury patients and their correlation with neuropsychometric tests: a 2-year follow-up study. J Head Trauma Rehabil 25:3142.

Langlois JA, Rutland-Brown W, Thomas KE (2005): The incidence of traumatic brain injury among children in the United States: differences by race. J Head Trauma Rehabil 20:229-238.

-Levin HS, Benavidez D, Verger-Maestre K, Perachio N, Song J, Mendelsohn D, et al (2000): Reduction of corpus callosum growth after severe traumatic brain injury in children. Neurology 54:647-653.

Levin HS, Wilde EA, Chu Z, Yallampalli R, Hanten G, Li X, et al (2008): Diffusion tensor imaging in relation to cognitive and function outcome of traumatic brain injury in children. J Head Trauma Rehabil 23:197208.

Levin HS, Williams DH, Valastro M, Eisenberg HM, Crofford MJ, Handei SF (1990): Corpus callosal atrophy following closed head injury: detection with magnetic resonance imaging. J Neurosurg 73:77-81.

Little DM, Kraus MF, Joseph J, Geary EK, Susmaras T, Zhou XJ, et al (2010): Thalamic integrity underlies executive dysfunction in traumatic brain injury. Neurology 74:558564.

Löbel U, Sedlacik J, Güllmar D, Kaiser WA, Reichenbach JR, Mentzel HJ (2009): Diffusion tensor imaging: the normal evolution of ADC, RA, FA, and eigenvalues studied in multiple anatomical regions of the brain. Neuroradiology 51:253-263.

MacDonald CL, Dikranian K, Bayly P, Holtzman D, Brody D (2007): Diffusion tensor imaging reliably detects experimental traumatic axonal injury and indicates approximate of injury. J Neurosci 27:11869-11876.

MacDonald CL, Dikranian K, Song SK, Bayly P, Holtzman DM, Brody DL (2007): Detection of traumatic axonal injury with diffusion tensor imaging in a mouse model of traumatic brain injury. Exp Neurol 205:116-131.

Madigan NK, DeLuca J, Diamond BJ, Tramontano G, Averiall A (2000): Speed of information processing in traumatic brain injury: modality-specific factors. J Head Trauma Rehabil 15:943-956.
Markham JA, Herting MM, Luszpak AE, Juraska JM, Greenough WT (2009): Myelination of the corpus callosum in male and female rats following complex environment housing during adulthood. Brain Res 1288:9-17.

- Mayer AR, Ling J, Mannell MV, Gasparovic C, Phillips JP, Doezema D, et al (2010): A prospective diffusion tensor imaging study in mild traumatic brain injury. Neurology 74 : 643-650.

-Merkley TL, Bigler ED, Wilde EA, McCauley SR, Hunter JV, Levin HS (2008): Diffuse changes in cortical thickness in pediatric moderateto-severe traumatic brain injury. J Neurotrauma 25:1343-1345.

Morey RA, Selgrade ES, Wagner HR 2nd, Huettel SA, Wang L, McCarthy G (2010): Scanrescan reliability of subcortical brain volumes derived from automated segmentation. Hum Brain Mapp, E-pub ahead of print.

Mori S, Crain BJ, Chacko VP, Vanzijl PC (1999): Three-dimensional tracking of axonal projections in the brain by magnetic resonance imaging. Ann Neurol 45:265-269.

Mori S, Zhang J (2006): Principles of diffusion tensor imaging and its applications to basic neuroscience research. Neuron 51:527-539.

Mukherjee P, Berman JI, Chung SW, Hess CP, Henry RG (2008): Diffusion tensor MR imaging and fiber tractography: theoretic underpinnings. Am J Neuroradiol 29:632-641.

- Nakayama N, Okumura A, Shinoda J, Yasokawa YT, Yoshimura SI, Iwama T (2006): Evidence for white matter disruption in traumatic brain injury without macroscopic lesions. J Neurol Neurosurg Psychiatry 77:850-855.

Netsch T, Rösch P, van Muiswinkel A, Weese J (2001): Towards real-time multi-modality 3-D medical image registration. ICCV, Vancouver, vol 1, pp 718-725.

Newcombe VFJ, Williams GB, Nortje J, Bradley PG, Harding SG, Smielewski P, et al (2007): Analysis of acute traumatic axonal injury using diffusion tensor imaging. Br J Neurosurg 21:340-348.

Niogi SN, Mukherjee P, Ghajar J, Johnson C, Koslter RA, Sarkar R, et al (2008): Extent of microstructural white matter injury in postconcussive syndrome correlates with impaired cognitive reaction time: a 3-T diffusion tensor imaging study of mild traumatic brain injury. Am J Neuroradiol 29:967-973.

-Pannek K, Mathias JL, Bigler ED, Brown G, Taylor JD, Rose S (2010): An automated strategy for the delineation and parcellation of commissural pathways suitable for clinical populations utilising high angular resolution diffusion imaging tractography. Neuroimage 50:1044-1053.

- Parizel PM, Ozsarlak O, van Goethem JW, van den Hauwe L, Dillen C, Verlooy J, et al (1998): Imaging findings in diffuse axonal injury after closed head trauma. Eur Radiol 8:960965 
Patel MD, Toussaint N, Charles-Edwards GD, \Smith DH, Meaney DF, Shull WH (2003): DifLin JP, Batchelor PG (2010): Distribution and fibre field similarity mapping of the human anterior commissure fibres by diffusion tensor imaging. MAGMA, E-pub ahead of print.

Povlishock JT, Katz DI (2005): Update of neuropathology and neurological recovery after traumatic brain injury. J Head Trauma Rehabil 20:76-94.

Ptak T, Sheridan RL, Rhea JT, Gervasini AA, Yun JH, Curran MA, et al (2003): Cerebral fractional anisotropy score in trauma patients: a new indicator of white matter injury after trauma. Am J Radiol 181:1401-1407.

- Reeves TM, Phillips LL, Povlishock JT (2005): Myelinated and unmyelinated axons of the corpus callosum differ in vulnerability and functional recovery following traumatic brain injury. Exp Neurol 196:126-137.

Rugg-Gunn FJ, Symms MR, Barker GJ, Greenwood R, Duncan JS (2001): Diffusion imaging shows abnormalities after blunt head trauma when conventional magnetic resonance imaging is normal. J Neurol Neurosurg Psychiatry 70:530-533.

- Salmond CH, Menon DK, Chatfield DA, William GB, Pena A, Sahakian BJ, et al (2006): Diffusion tensor imaging in chronic head injury survivors: correlations with learning and memory indices. Neuroimage 29:117124.

-Sidaros A, Engberg AW, Sidaros K, Liptrot MG, Herning M, Petersen P, et al (2008): Diffusion tensor imaging during recovery from severe traumatic brain injury and relation to clinical outcome: a longitudinal study. Brain 131:559-572.

- Sidaros A, Skimminge A, Liptrot MG, Sidaros K, Engberg AW, Herning M, et al (2009): Longterm global and regional brain volume changes following severe traumatic brain injury: a longitudinal study with clinical correlates. Neuroimage 44:1-8.

Sled JG, Zijdenbos AP, Evans AC (1998): A nonparametric method for automatic correction of intensity nonuniformity in MRI data. IEEE Trans Med Imaging 17:87-97. fuse axonal injury in head trauma. J Head Trauma Rehabil 18:307-316.

- Tanriverdi T, Olivier A, Poulin N, Andermann F, Dubeau F (2009): Long-term seizure outcome after corpus callosotomy: a retrospective analysis of 95 patients. J Neurosurg 110: 332-342.

Taylor HG (2004): Research on outcomes of pediatric traumatic brain injury: current advances and future directions. Dev Neuropsychol 25:199-225.

Teasdale G, Jennett B (1974): Assessment of coma and impaired consciousness: a practical scale. Lancet ii:81-84.

Thurman DJ, Coronado V, Selassie A (2007): The epidemiology of TBI: implications for public health; in Zasler ND, Katz DI, Zafonte RD (eds): Brain Injury Medicine. New York, Demos Medical, pp 45-55.

Tomaiuolo F, Carlesimo GA, Paola MD, Petrides M, Fera F, Bonanni R, et al (2004): Gross morphology and morphometric sequelae in the hippocampus, fornix, and corpus callosum of patients with severe non-missile traumatic brain injury without macroscopically detectable lesions: a $\mathrm{T}_{1}$-weighted MRI study. J Neurol Neurosurg Psychiatry 131:13141322.

Turken AU, Whitfield-Gabrieli S, Bammer R, Baldo JV, Dronkers NF, Gabrieli JDE (2008): Cognitive processing speed and the structure of white matter pathways: convergent evidence from normal variation and lesion studies. Neuroimage 42:1032-1044.

van der Zijden JP, van der Toorn A, van der Marel K, Dijkhuizen RM (2008): Longitudinal in vivo MRI of alterations in perilesional tissue after transient ischemic stroke in rats. Exp Neurol 212:207-212.

Wang J, Bakhadirov K, Devous MD, Adbdi H, McColl R (2008): Diffusion tensor tractography of traumatic diffuse axonal injury. Arch Neurol 65:619-626.
-Wang S, Wu EX, Qiu D, Leung LH, Lau HF, Khong PL (2009): Longitudinal diffusion tensor magnetic resonance imaging study of radiation-induced white matter damage in a rat model. Cancer Res 69:1190-1198.

-Wilde EA, Chu Z, Bigler ED, Hunter JV, Fearing MA, Hanten GR, et al (2006): Diffusion tensor imaging in the corpus callosum in children after moderate-to-severe traumatic brain injury. J Neurotrauma 23:1412-1426.

-Wilde EA, McCauley SR, Hunter JV, Bigler ED, Chu Z, Wang J, et al (2008): Diffusion tensor imaging of acute mild traumatic brain injury in adolescents. Neurology 70:948-955.

-Wu TC, Wilde EA, Bigler ED, Yallampalli R, McCauley SR, Troyanskaya M, et al (2010): Evaluating the relationship between memory functioning and cingulum bundles in acute mild traumatic brain injury using diffusion tensor imaging. J Neurotrauma 27:303-307.

-Xu J, Rasmussen I, Lagopoulos J, Haberg A (2007): Diffuse axonal injury in severe traumatic brain injury visualized using high-resolution diffusion tensor imaging. J Neurotrauma 24:753-765.

- Yamada K, Sakai K, Akazawa K, Yuen S, Nishimura T (2009): MR tractography: a review of its clinical applications. Magn Reson Med Sci 8:165-174.

Yeates KO, Taylor HG, Drotar D, Wade SL, Klein S, Stancin T, et al (1997): Preinjury family environment as a determinant of recovery from traumatic brain injury in school-age children. J Int Neuropsychol Soc 3:617-630.

-Yuan W, Holland SK, Schmithorst VJ, Walz NC, Cecil KM, Jones BV, et al (2007): Diffusion tensor MR imaging reveals persistent white matter alteration after traumatic brain injury experienced during early childhood. Am J Neuroradiol 28:1919-1925.

Zhang L, Thomas KM, Davidson MC, Casey BJ, Heier LA, Ulug AM (2005): MR quantitation of volume and diffusion changes in the developing brain. Am J Neuroradiol 26:45-49. 